\title{
Fundamental limitations on Earth-like planet detection with ELTs
}

\author{
Céline Cavarroc ${ }^{1}$, Anthony Boccaletti ${ }^{1}$, Pierre Baudoz ${ }^{2}$, \\ Thierry Fusco ${ }^{2}$ and Daniel Rouan ${ }^{1}$ \\ ${ }^{1}$ Observatoire de Paris-Meudon, Meudon, France \\ email: celine.cavarroc@obspm.fr \\ ${ }^{2}$ ONERA, Châtillon, France
}

\begin{abstract}
A forthcoming step in the study of extrasolar planetary systems is the direct detection and characterization of Earth-like planets. An asset of the ELTs in that context is their very high angular resolution and their collecting area. The luminosity ratio between a terrestrial planet and its star $\left(10^{-10}\right)$ is such an ambitious goal that a thorough study needs to be carried out. We started with a simple analysis of the fundamental limitations for the detection of extraterrestrial planets with ELTs. Here, we considered an extreme adaptive optics device upstream of a perfect coronagraph. Even with high Strehl ratios, the coronagraphic halo level is only $10^{-6}$ to $10^{-7}$ at typical exo-Earth angular distances. A calibration device is therefore mandatory to reach the contrast between a terrestrial planet and its star in the near infra-red. We considered a simple but realistic model taking into account dynamic aberrations left uncorrected by the adaptive optics system, static aberrations of optical system and differential static aberrations due to the calibration channel itself. Numerical simulations prove that, after the calibration, the limitations are set by the static aberrations which cannot be neglected anymore.
\end{abstract}

Keywords. instrumentation: high angular resolution, adaptive optics - methods: numerical, analytical - (stars:) planetary systems

\section{Introduction}

The ultimate goal in the study of Extra-solar planets is direct imaging and characterization of Earth-like planets. In that context, the Extremely Large Telescopes (ELTs) are very promising.

The detection of Earth-like planet in the visible or in the near infra-red requires a contrast of $10^{-10}$ close to the star. To reach such a high contrast, we consider a coronagraph associated with an extreme adaptive optics device. However, it is not sufficient to detect an Earth-like planet and a calibration channel is needed to enhance the performance of the system.

The first section presents the model and the hypotheses we made to simulate it. The second one presents the limitations imposed by aberrations on the speckle noise.

\section{Model}

The objective of the paper is to study only the effects of the aberrations on the performance of a classical system and the limitations they impose. We consider an ELT associated with a coronagraphic device and a calibration channel downstream the coronagraph (Fig. 1). To reduce the speckle noise, the calibration is done simultaneously. We do not assume any particular system of calibration for sake of generality (different types can be assumed: spectral calibration (Racine 1999), calibration of the polarization (Baba and Murakamo 2003) or the use of the properties of coherence of the speckles with the 


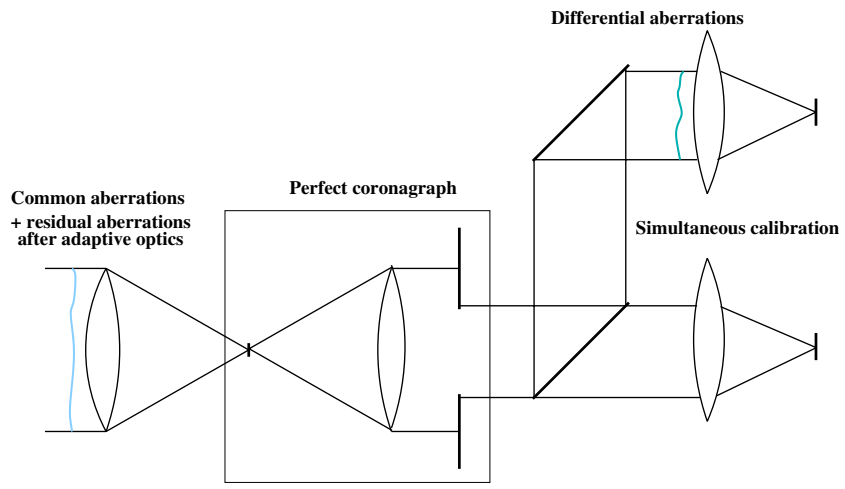

Figure 1. Generic concept of the studied system with, from left to right, the telescope pupil, the focal plane, the relayed optics with the Lyot stop and differential optics. The intensity distribution at a given plane is defined by the Fourier transform of the upstream plane. Phase aberrations are included in the telescope pupil and in the pupil after the beam splitter.

star (Guyon 2004, Baudoz et al. 2005) and we make the optimistic assumption that the planet is shown in one channel and not in the other.

Besides, we assume a monolithic entrance pupil (without segmentation) and a perfect coronagraph, i.e. a coronagraph, which removes all the coherent light perfectly.

We also include static aberrations of the instrument: common aberrations upstream of the coronagraph $\delta_{C}$ and differential aberrations $\delta_{N C}$ due to the difference of the optical path between the two channels and dynamic aberrations due to the imperfect correction of adaptive optics $\phi$.

In that condition the residual intensity $I_{r}$ of a short exposure after subtracting the two channels takes the following form (Cavarroc et al. 2006), with $\Pi$ the entrance pupil:

$$
\begin{aligned}
I_{r} & =2 \Im\left((\widehat{\Pi} \star \widehat{\phi}) \cdot\left(\widehat{\Pi} \star \widehat{\phi} \star \widehat{\delta_{N C}}\right)^{*}\right) \\
& +2 \Im\left((\widehat{\Pi} \star \widehat{\phi}) \cdot\left(\widehat{\Pi} \star \widehat{\delta_{C}} \star \widehat{\delta_{N C}}\right)^{*}\right) \\
& +2 \Im\left(\left(\widehat{\Pi} \star \widehat{\delta_{C}}\right) \cdot\left(\widehat{\Pi} \star \widehat{\phi} \star \widehat{\delta_{N C}}\right)^{*}\right) \\
& +2 \Im\left(\left(\widehat{\Pi} \star \widehat{\delta_{C}}\right) \cdot\left(\widehat{\Pi} \star \widehat{\delta_{C}} \star \widehat{\delta_{N C}}\right)^{*}\right)
\end{aligned}
$$

with $\Im$ the imaginary part of the expression, ${ }^{*}$ the conjugate and $\widehat{.}$ the Fourier transform. $\star$ represents the convolution product. For long exposure, it is shown that the level of detection is only given by:

$$
\sigma_{\theta}\left(<I_{r}>_{t}\right)=\sigma_{\theta}\left(2 \Im\left[\left(\widehat{\Pi} \star \widehat{\delta_{C}}\right) \cdot\left(\widehat{\Pi} \star \widehat{\delta_{C}} \star \widehat{\delta_{N C}}\right)^{*}\right]\right)
$$

This detectability only depends on static aberrations, linearly according to differential aberrations and quadratically according to common aberrations.

\section{Results of numerical simulations}

\subsection{Hypotheses}

In this section, we only consider the speckle noise for a $30 \mathrm{~m}$ and a $100 \mathrm{~m}$ telescope and its effect on the detectability level, neglecting the photon noise.

We assume an extreme adaptive optics system: it provides a Strehl ratio of about $98 \%$. The atmospheric phase screens are generated from analytical expressions (Rigaut et al. 
1998). We consider a pyramidal wavefront sensor (it better corrects low frequencies) with 200 actuators across the pupil diameter. The seeing is $0.75 \operatorname{arcsec}$ at $\lambda=0.5 \mu \mathrm{m}$. The imaging wavelength is $1.63 \mu \mathrm{m}$. We assume a 5 th magnitude star and a loop frequency of $2.5 \mathrm{kHz}$. The power spectrum density (PSD) includes fitting errors, the servo-lag and the photon noise of the wavefront sensor.

The PSDs of static aberrations varies like $f^{-2}$ (Duparré et al. 2002) but are attenuated at low frequencies to mimic a phase diversity algorithm. We consider that this correction is done in the instrument so the shape of the PSD of static aberrations is identical for a $30 \mathrm{~m}$ and a $100 \mathrm{~m}$ telescopes.

\subsection{Limitations due to static aberrations}

Equation 2.2 proves that the fundamental limit is only set by static aberrations. So the choice of the shape of the power spectrum density is essential: for a pure variation in $f^{-2}$ with the same error budget, the final level would be better at high frequencies but worse in the region of interest.

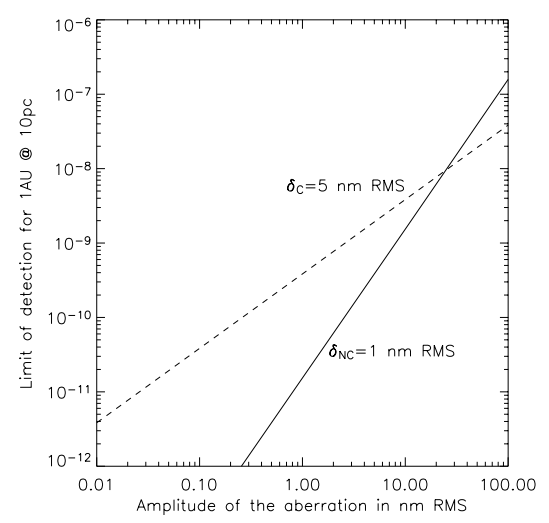

Figure 2. Detectability at $5 \sigma$ for an angular separation of $1 \mathrm{AU}$ at $10 \mathrm{pc}$ for a long exposure. The solid line stands for the impact of the common static aberration when fixing the differential aberrations and conversely for the dashed line. Values are valid for $30 \mathrm{~m}$ and $100 \mathrm{~m}$ telescopes.

Fig. 2 shows the influence of the amplitude of static aberrations for an angular separation of $0.1^{\prime \prime}(9 \lambda / D$ for a diameter of $30 \mathrm{~m}$ and $30 \lambda / D$ for a diameter of $100 \mathrm{~m})$ from $10 \mathrm{pm}$ to $10 \mathrm{~nm}$. The effect of the common and differential aberrations is clearly different: the detectability varies linearly with the differential aberrations and quadratically with common aberrations which is coherent with eq. 2.2 .

Therefore, to reach the desired contrast of $10^{-10}$ at $0.1^{\prime \prime}$, for common aberrations of $5 \mathrm{~nm}$, we have to control differential aberrations to $0.2 \mathrm{~nm}$ and, conversely, for differential aberrations of $1 \mathrm{~nm}$ the common aberrations must be reduced under $0.3 \mathrm{~nm}$ RMS.

Common aberrations, i.e. aberrations upstream of the coronagraph, are dominant and must be reduced as far as possible.

In the next section we will consider common static aberrations of $5 \mathrm{~nm}$ and differential static aberrations of $0.2 \mathrm{~nm}$.

\subsection{Detectability}

The objective of this section is to study the gain brought by differential imaging. For that purpose, we compare detectabilities for different images:

- for a coronagraphic image of an instantaneous exposure, 
- for an instantaneous image after subtraction,

- for a long exposure after subtraction of the calibration image. The long exposure is obtained by adding several hundreds of images obtained from decorrelated AO phase screens.
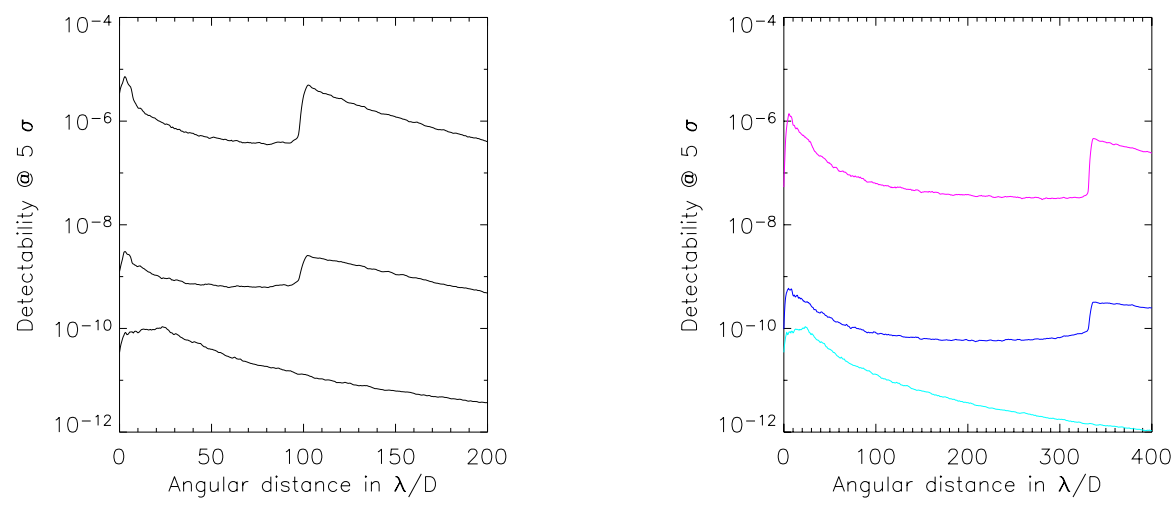

Figure 3. Detectability at $5 \sigma$. The upper curve corresponds to the detectability for a short exposure in a direct imaging mode, the middle curve for a short exposure after subtraction and the lower for a long exposure after subtraction. Left figure is for a $30 \mathrm{~m}$ telescope while right one is for a $100 \mathrm{~m}$ telescope.

On a short exposure, the detectability is determined by the AO correction, even after the simultaneous subtraction but, by subtracting, we gain more than a factor 1000 on a short exposure. For a 30m telescope, the level of the direct image is almost 10 times worse than for the $100 \mathrm{~m}$ which is critical for the photon noise.

\section{Conclusion}

The assumptions we made in this study are quite simple: there is no segmentation of the telescope pupil, the coronagraph is perfect. The model of the adaptive optics system is quite simple and we considered static aberrations before and after the coronagraph to take into account the imperfect calibration.

In spite of that, detecting an Earth-like planet at $0.1^{\prime \prime}$ from a Sun like star even with a $100 \mathrm{~m}$ telescope requires a challenging budget of static aberrations.

A complete study of the system must be performed to determine precisely the capability of detection of such telescopes.

\section{References}

Baba, N. \& Murakami, N. 2003, PASP 115, 1363

Baudoz, P., Boccaletti, A., Baudrand, J. \& Rouan, D. 2005, IAUC 200, in press

Cavarroc, C. and Boccaletti, A., Baudoz, P., Fusco, T. \& Rouan, D. 2006, A\&A 447, 397

Duparré, A., Ferre-Borrull, J., Gliech, S., Notni, G., Steinert, J. \& Bennett, J.M. 2002, AO 41, 154

Guyon, O. 2004, ApJ 615, 562

Racine, R., Walker, G.A.H., Nadeau, D., Doyon, R. \& Marois, C. 1999, PASP 111, 587

Rigaut, F., Salmon, D., Arsenault, R., Thomas, J., Lai, O., Rouan, D., Véran, J.P., Gigan, P., Crampton, D., Fletcher, J.M., Stilburn, J., Boyer, C. \& Jagourel, P. 1998, PASP 110, 152 


\section{Discussion}

STAPELFELDT: Just a comment: The $0.2 \mathrm{~nm}$ requirement you found for non-common path wavefront errors will be difficult to achieve. The best solution would be to do wavefront sensing in the science focal plane, instead of in a separate wavefront reference channel. 